\title{
ANSIEDAD, DEPRESIÓN, CALIDAD DE VIDA Y CONSUMO DE TABACO EN PERSONAS CON DIABETES MELLITUS TIPO 2
}

\author{
ANXIETY, DEPRESSION, QUALITY OF LIFE AND TOBACCO CONSUMPTION IN PEOPLE WITH TYPE 2 \\ DIABETES MELLITUS
}

Daniel Pech-Puebla, Jennifer Lira-Mandujano, Sara E. Cruz-Morales y Leonardo Reynoso-Erazo

\author{
Universidad Nacional Autónoma de México
}

\begin{abstract}
Es importante evaluar las diferencias entre ansiedad y depresión, y la asociación entre fumar y calidad de vida de fumadores y no fumadores mexicanos con Diabetes Mellitus (DM). Objetivo. Evaluar las diferencias entre fumadores y no fumadores con Diabetes Mellitus tipo 2 (DM2) en ansiedad, depresión y calidad de vida; evaluar si la ansiedad y depresión moderan la asociación entre calidad de vida y consumo de tabaco; y evaluar el nivel de predicción de la calidad de vida hacia el fumar. Metodología. 44 personas fumadoras y no fumadoras con DM2. Se recopiló información del patrón de consumo, dependencia a la nicotina, ansiedad, depresión y calidad de vida. Resultados. La ansiedad como rasgo y la depresión como rasgo y estado moderan la asociación entre el patrón de consumo y calidad de vida. Conclusiones. Las personas fumadoras con DM tuvieron más síntomas de depresión y ansiedad, y se vieron más afectadas en calidad de vida. La ansiedad y depresión moderan la asociación entre calidad de vida y dependencia a la nicotina. Se resalta la importancia de tomar en cuenta estos factores moderadores en las intervenciones para dejar de fumar y el mantenimiento de la abstinencia de tabaco en personas con DM2.
\end{abstract}

Palabras clave: ansiedad, calidad de vida, depresión, diabetes mellitus, tabaco

It is important to evaluate the differences in anxiety and depression, and the association between smoking and quality of life between Mexican smokers and non-smokers with Diabetes Mellitus (DM). Objective. To evaluate the differences between smokers and non-smokers with type 2 Diabetes Mellitus (T2DM) in anxiety, depression and quality of life; assess whether anxiety and depression moderate the association between quality of life and tobacco use; and evaluate the level of prediction of the quality of life towards tobacco consumption. Methodology. 44 smokers and non-smokers with T2DM. Information on the pattern of consumption, nicotine dependence, anxiety, depression, and quality of life was collected. Results. Anxiety as a trait and depression as a trait and state moderate the association between the pattern of consumption and quality of life. Conclusions. Smokers with DM had more symptoms of depression and anxiety and were more affected in quality of life. Anxiety and depression moderate the association between quality of life and nicotine dependence. The importance of taking these moderating factors into account in interventions for stop smoking and the maintenance of tobacco abstinence in people with T2DM is highlighted.

Keywords: anxiety, depression, diabetes mellitus, quality of life, tobacco.

Daniel Pech Puebla, Facultad de Estudios Superiores Iztacala, Universidad Nacional Autónoma de México, Tlalnepantla, Estado de México, México, https://orcid.org/0000-0003-2044-017X

Jennifer Lira Mandujano, Facultad de Estudios Superiores Iztacala, Universidad Nacional Autónoma de México, Tlalnepantla, Estado de México, México, correspondencia liramjenn@comunidad.unam.mx, https://orcid.org/0000-0002-7331-4735

Sara Eugenia Cruz Morales, Facultad de Estudios Superiores Iztacala, Universidad Nacional Autónoma de México, Tlalnepantla, Estado de México, México.

Leonardo Reynoso Erazo, Facultad de Estudios Superiores Iztacala, Universidad Nacional Autónoma de México, Tlalnepantla, Estado de México, México, https://orcid.org/0000-0001-9793-1431 
La Diabetes Mellitus (DM) es un problema de salud de relevancia internacional que se asocia con aproximadamente cuatro millones de muertes y representa una fuerte inversión económica con respecto a los costos directos de atención médica atribuibles a la DM (International Diabetes Federation [IDF], 2017). La World Health Organization (2018) menciona que la DM es una enfermedad crónica que se clasifica en diabetes mellitus tipo uno (DM1), donde hay deficiencia en la producción de la insulina y diabetes mellitus tipo dos (DM2), donde el cuerpo no es capaz de aprovechar la insulina de manera adecuada. La IDF (2017) menciona que existen aproximadamente 425 millones de personas en el mundo de entre 20 y 79 años con DM, y que para el año 2045 habrá aproximadamente 629 millones de personas con DM. En México, la prevalencia de personas adultas con DM aumentó del $9.2 \%$ en 2012 a $9.4 \%$ en el año 2016 (Instituto Nacional de Salud Pública [INSP], 2016). En el 2018, hubo un total de 3,279 nuevos casos DM1 y una incidencia de 5.95 (tasa por 100,000 habitantes), mientras que para la DM2 el número de casos nuevos fue de 425,345 y una incidencia de 411.85 (Dirección General de Epidemiología, 2018)

Diferentes asociaciones en el mundo (IDF, 2012; Organización mundial de la Salud [OMS], 2017) y en México (Secretaría de Salud [SSA], 2015), señalan que el tratamiento de la DM debe estar enfocado a una buena alimentación, a promover la actividad física, mantener revisiones médicas periódicas, realizar mediciones de glucosa en sangre, recibir educación sobre la diabetes, sus consecuencias en la salud y sus cuidados, buen apego a la medicación en caso de ser necesaria, e incluir estrategias para eliminar el consumo de alcohol y tabaco.

La DM2 se caracteriza por la hiperglucemia, y el impacto principal de esta enfermedad está en las complicaciones vasculares, derivadas de la concentración elevada de glucosa en la sangre. Las consecuencias en la salud por la falta de control de la DM2 se presentan a nivel microvasculares (retinopatía diabética, nefropatía diabética, y neuropatía diabética), macro vasculares (ateroesclerosis, cardiopatía isquémica y eventos vascular cerebrales) y riesgos de cáncer en páncreas (Pereira et al., 2016; Maric-Bilkan, 2017; OMS, 2017), las cuales han mostrado un incremento importante en los últimos años. Los mecanismos que llevan al daño vascular son múltiples e implican diversas alteraciones metabólicas y estructurales como la generación de productos finales de la glicosilación avanzada, activación anormal de cascada de señales (tales como la protein quinasa C), mayor estrés oxidativo y la consiguiente disfunción endotelial (Valero et al., 2012). La DM2 acelera el desarrollo de aterosclerosis y de la enfermedad microvascular (Pereira et al., 2016), y conlleva una reducción en la expectativa de vida de tres hasta 17 años (Huo et al., 2016; Ou, Yang, Wang, Hwang, \& Wu, 2016; Walker et al., 2018).

Además del impacto de la DM en la salud, los factores emocionales como ansiedad y depresión también juegan un papel importante en personas con DM. Con respecto a esto, diferentes estudios (Fabián \& Cobo, 2007; Roy \& Lloyd, 2012; Tovilla-Zárate et al., 2012) muestran que hay una asociación importante entre la ansiedad, depresión y la DM, y que tener el diagnóstico de DM representa mayor riesgo de tener síntomas de depresión, lo cual dificulta la adherencia al tratamiento y los cuidados de la enfermedad, lo que incrementa la probabilidad de consumir tabaco para modular la sintomatología de ansiedad y de depresión, y adicionalmente, dichos factores dificultan el cese de consumo de tabaco. Además, un estudio realizado por Osme et al. (2012), mostró que en personas fumadoras con DM2, la prevalencia de síntomas de depresión y ansiedad son del $30 \%$ y 50 \% respectivamente, y la prevalencia de síntomas de ansiedad es mayor en comparación con quienes no fuman, con $19 \%$ y $4.3 \%$ respectivamente. 
Asimismo, el consumo de tabaco en personas con esta enfermedad representa un aumento en la probabilidad de riesgos en la salud, ya que además de las consecuencias asociadas al consumo de tabaco, como lo son diferentes tipos de cáncer, enfermedades respiratorias, y enfermedades cardiovasculares (National Institute on Drug Abuse [NIDA], 2019), las propiedades farmacológicas de la nicotina juegan un papel importante. En este sentido, en un estudio realizado por Tweed, Hsia, Lutfy, y Friedman (2012) se observó que la nicotina procedente de fumar cigarrillos incrementa los niveles circulatorios de cortisol, hormona del crecimiento y prolactina en fumadores hombres, el consumo de cigarrillos estimula la liberación de algunas hormonas de la adenohipófisis, como la adenocorticotropina (ACTH), hormona del crecimiento y vasopresina. En el reporte del cirujano general de los EUA del 2014 (U.S. Department of Health and Human Services, 2017) se menciona que el fumar puede contribuir al desarrollo de resistencia a la insulina, y el Centers for Disease Control and Prevention (CDC) (2017) menciona que la nicotina provoca resistencia a la insulina, aumenta la prevalencia de dislipemias, y eleva el nivel de glucosa en sangre, lo que incrementa el riesgo de padecer cardiopatías, amputaciones, nefropatías, retinopatías y neuropatías.

Diferentes estudios mencionan que existe un importante porcentaje de personas con DM que consumen tabaco, el cual oscila del 3.9\% al 28\% (Albaroodi, Syed, Shafie, Awaisu, \& Lajs, 2014; Gilliani, Aziz, Bashir, Mustafa, \& Fang, 2017; Stoddard, He, \& Schillinger, 2010), por lo tanto, la American Diabetes Association (ADA) (2018) sugiere que el cese de consumo de tabaco debe ser parte de los estándares del tratamiento de la DM.

Además de los efectos en la salud por el consumo de tabaco, las personas fumadoras presentan mayores síntomas depresivos, hospitalizaciones, trastornos mentales (Pizzo, Matsuo, \& Vargas, 2009), y menor calidad de vida relacionada con la salud (De Miguel Díez et al., 2010; Džubur et al., 2014; López-Nicolás, Trapero-Bertran, \& Muñoz, 2018; Pizzo et al., 2009) en comparación con quienes no fuman, y el padecer enfermedades que se encuentren asociadas con el consumo de tabaco también afecta a la calidad de vida (López-Nicolás et al., 2018).

Con respecto a las personas fumadoras con DM, se ha observado que los factores que afectan la calidad de vida en personas con DM son el tener el diagnóstico de DM (Grandy, Chapman, Fox, \& SHIELD Study Group, 2008), el momento de diagnóstico de la DM, el consumo de alcohol y tabaco (Oliveira et al., 2017), y el tener mayor tiempo de diagnóstico, un alto Índice de Masa Corporal (IMC) (Akinci et al., 2008; Kalda, Rätsep, \& Lember, 2008), mientras que las áreas más perjudicadas en la calidad de vida son el funcionamiento físico, el funcionamiento mental (Ahmed, Tomalika, Rhaman, Momtaz, \& Haque, 2018), y la salud en general (Alencar et al., 2019; Mokhtari, Gheshlagh, \& Kurdi, 2019). Asimismo, en personas con DM2, la sintomatología depresiva es mayor en comparación con quienes no tienen DM2 (Grandy et al., 2008), por lo que, debido a las complicaciones de salud y los problemas de salud mental, las personas con DM tienen una moderada calidad de vida (Mokhtari et al., 2019).

A partir de los estudios revisados se evidencia que, en personas fumadoras, la sintomatología de ansiedad y depresión son mayores y afectan a la calidad de vida, y que los síntomas de depresión, el tiempo y momento de diagnóstico, así como el alto IMC son factores que se asocian con un deterioro en la calidad de vida en personas con DM. De acuerdo con la revisión de la literatura, poco se sabe acerca de la relación que existe entre ansiedad, depresión, calidad de vida, y consumo de tabaco, cuando además de tener el diagnóstico de DM se consume tabaco, por lo tanto, el objetivo general de este estudio fue evaluar las diferencias que existen entre 
fumadores y no fumadores con respecto a la ansiedad, la depresión en sus dimensiones rasgo y estado, y la calidad de vida. Como objetivos específicos se planteó evaluar si la ansiedad y depresión como rasgo y estado moderan la asociación entre la calidad de vida y el consumo de tabaco en personas fumadoras, así como evaluar el nivel de predicción que tiene la calidad de vida con respecto al consumo de tabaco, de acuerdo con los niveles de ansiedad y depresión en rasgo y estado en fumadores con DM2.

\section{Método}

\section{Diseño y participantes}

Se utilizó un enfoque cuantitativo, con alcance correlacional, y un diseño transversal no experimental con muestreo no probabilístico.

44 personas con DM2 que aceptaron participar en el estudio, de los cuales el 50\% consumían tabaco (ver Tabla 1).

Tabla 1

Características de la muestra

\begin{tabular}{|c|c|c|c|}
\hline & No fumadores & Fumadores & Todos los participantes \\
\hline & $n(\%)$ & $n(\%)$ & $n(\%)$ \\
\hline Hombres & $8(36.4)$ & $9(40.9)$ & $17(38.6)$ \\
\hline \multirow[t]{2}{*}{ Mujeres } & $14(63.6)$ & $13(59.1)$ & $27(61.4)$ \\
\hline & $\bar{X}(S D)$ & $\bar{X}(S D)$ & $\bar{X}(S D)$ \\
\hline Edad & $44.91(8.56)$ & $45(6.81)$ & $44.95(7.64)$ \\
\hline $\begin{array}{l}\text { Años con el } \\
\text { diagnóstico de } \\
\text { DM2 }\end{array}$ & $5.32(3.74)$ & $5.59(2.98)$ & $5.45(3.35)$ \\
\hline $\begin{array}{l}\text { Inicio de consumo } \\
\text { de tabaco }\end{array}$ & N/A & $19.68(6.09)$ & N/A \\
\hline $\begin{array}{l}\text { Años de consumo } \\
\text { regular de tabaco }\end{array}$ & N/A & $16.18(9.33)$ & N/A \\
\hline $\begin{array}{l}\text { Intentos de cese } \\
\text { de consumo de } \\
\text { tabaco }\end{array}$ & N/A & $2.27(2)$ & N/A \\
\hline $\begin{array}{l}\text { Glucosa en sangre } \\
(\mathrm{mg} / \mathrm{dl})\end{array}$ & $139.81 \mathrm{mg} / \mathrm{dl}(8.32)$ & $131.95 \mathrm{mg} / \mathrm{dl}(13.63)$ & 135.88 mg/dl (11.85) \\
\hline $\begin{array}{l}\text { Monóxido de } \\
\text { carbono (COppm) }\end{array}$ & N/A & 6.86 COppm (5.84) & N/A \\
\hline
\end{tabular}

Criterios de inclusión: edad entre 30 y 60 años, saber leer y escribir, y firmar el consentimiento informado, el cual tuvo como objetivo proporcionar información acerca de los 
objetivos del estudio, sus consideraciones éticas, y solicitar el consentimiento escrito sobre la participación en el estudio.

Criterios de exclusión: tener el diagnóstico de alguna enfermedad crónico-degenerativa diferente a DM o algún trastorno psiquiátrico, utilizar medicamento de reemplazo de nicotina, mujeres embarazadas, y tener consumo de riesgo de alguna sustancia diferente a tabaco de acuerdo con el ASSIST (OMS, 2011).

\section{Instrumentos}

Entrevista inicial: tiene como objetivo recopilar de datos sociodemográficos, de la diabetes e historia de consumo de tabaco (adaptada para población con DM a partir de la entrevista de Lira-Mandujano, 2009).

Línea Base Retrospectiva (LIBARE; Robinson, Sobell, Sobell, \& Leo, 2014): tiene como objetivo conocer el patrón de consumo de los participantes, con respecto a la frecuencia e intensidad en los 30 días previos a la evaluación. Cuenta con un coeficiente de confiabilidad test-retest .75 a .96 (adaptado a población mexicana por Lira-Mandujano, 2009).

Escala de Dependencia a los Cigarros (EDC; Etter, Le Houezec, \& Perneger, 2003): tiene como objetivo evaluar el nivel de dependencia a la nicotina, a través de 12 reactivos, a partir de criterios del DSM-IV y la CIE-10. Cuenta con un coeficiente de consistencia interna por alfa de Cronbach de .85 y coeficiente de validez test-retest de .83 .

Inventario de Ansiedad Rasgo-Estado (IDARE; Spielberger \& Díaz, 2002): tiene como objetivo evaluar la ansiedad en las dimensiones rasgo (cómo se siente la persona normalmente) y estado (cómo se siente la persona en el momento de la evaluación), y está constituido por 40 reactivos. Cuenta con un coeficiente alfa de Cronbach de .91 para la dimensión de rasgo y de .92 para la dimensión de estado (Galicia \& Segundo, 2007).

Inventario de Depresión Rasgo-Estado (IDERE; Martín, Grau, Ramírez, \& Grau, 2001): tiene como objetivo evaluar los niveles de depresión como rasgo y estado, y se compone por 42 reactivos. Cuenta con buena validez de criterio y convergente, con coeficientes de correlación estadísticamente significativos de .556 y .683, para las dimensiones rasgo y estado, respectivamente (Martín-Carbonell et al., 2012).

Cuestionario Diabetes-39 (D-39; Boyer \& Earp, 1997): tiene como objetivo evaluar la calidad de vida de las personas con DM2, está compuesto por 39 reactivos, consta de cinco sub escalas: energía y movilidad (EyM), control de la diabetes (CDD), ansiedad y preocupación (AyP), sobrecarga social (SS), y funcionamiento sexual (FS). Proporciona un puntaje en cada sub escala así como un puntaje total (PT), que representan el porcentaje de afectación en la calidad de vida. La versión adaptada y validada para población mexicana cuenta con coeficiente de confiabilidad por alfa de Cronbach de .95 y un punto de corte de 29 , donde porcentajes menores al punto de corte en el total del cuestionario, así como en sus sub escalas representan una baja afectación en la calidad de vida y, por lo tanto, una buena calidad de vida (López-Carmona \& RodríguezMoctezuma, 2006). 


\section{Procedimiento}

Los datos fueron recopilados en la evaluación inicial del tratamiento para dejar de fumar para personas con DM2. Se reclutó a los participantes en la Ciudad y Estado de México, en diferentes centros de salud y por difusión general. Un psicólogo especialista en adicciones realizó una sola sesión de admisión y evaluación de forma individual con cada uno de los participantes, de duración de 60 minutos, en la cual se les explicaba a las personas las características del tratamiento, y en caso de aceptar participar, se les pedía que leyeran y firmaran el formato de consentimiento informado, para posteriormente aplicar los instrumentos correspondientes. A aquellas personas fumadoras que aceptaron participar en el estudio se aplicaron de forma heteroaplicada los siguientes instrumentos: entrevista inicial, LIBARE, EDC, IDARE, IDERE, y D-39, mientras que a aquellas personas con DM que no fumaran, se les aplicaron de igual forma los siguientes instrumentos: entrevista inicial, IDARE, IDERE y D-39. A aquellas personas que no cumplieran con los criterios de inclusión o contaran con criterios de exclusión, se les proporcionó la intervención sin considerar sus datos para este estudio. Asimismo, aquellas personas que no contaran con los datos completos con respecto a los instrumentos aplicados tampoco fueron contempladas para los análisis en este estudio.

Los datos recopilados fueron capturados en una base de datos del programa ${ }^{\circledR}$ IBM SPSS Statistics 21, para ser analizados posteriormente.

\section{Análisis de datos}

Se realizaron análisis descriptivos de las variables: ansiedad, depresión, patrón de consumo, dependencia a la nicotina y calidad de vida para la muestra total, de los no fumadores y de los fumadores.

Para cumplir con el objetivo general, se realizaron pruebas t de Student para muestras independientes para evaluar las diferencias entre las medias de los no fumadores y los fumadores, con respecto a las variables de ansiedad y depresión como rasgo y estado, así como de cada una de las sub escalas de calidad de vida. Se realizaron análisis de correlación de Pearson para determinar las variables a incluir en los análisis de regresión para los objetivos específicos. Se realizaron análisis de regresión lineal múltiple a través del método de entrada forzada, con aquellos participantes fumadores, donde se incluyó en cada uno: una variable predictora (total o una sub escala del D-39), una segunda variable predictora (puntaje de ansiedad o depresión como rasgo o estado), una variable moderadora (variable predictora $1 \mathrm{X}$ variable predictora 2 ), y una variable dependiente (patrón de consumo o puntaje del EDC). Por último, se realizaron análisis de regresión lineal simple por niveles de ansiedad y depresión como rasgo y estado, incluyendo una variable predictora (total o una sub escala del D-39) y una variable dependiente (patrón de consumo o puntaje del EDC).

\section{Resultados}

De acuerdo con los datos descriptivos de la muestra, el patrón de consumo de los fumadores fue de $7.69(D E=10)$ cigarros diarios, con un puntaje medio de dependencia a la nicotina de 30.4 ( $D E=12.76)$, que equivale a $50.66 \%$. Como se muestra en la Tabla 2, los niveles de ansiedad y depresión como rasgo fueron medios, excepto por la depresión como rasgo del total de participantes, de los no fumadores y los fumadores, así como la ansiedad como estado de los 
fumadores, los cuales resultaron altos. Y con respecto a la calidad de vida, las áreas más afectadas fueron el CDD y la AyP en los tres grupos.

Tabla 2

Datos descriptivos de las variables

\begin{tabular}{lcccccc}
\hline & \multicolumn{2}{l}{ Todos los participantes } & \multicolumn{2}{c}{ No fumadores } & \multicolumn{2}{c}{ Fumadores } \\
\hline & $\bar{X}(S D)$ & Nivel & $\bar{X}(S D)$ & Nivel & $\bar{X}(S D)$ & Nivel \\
\hline AE & $41.36(9.30)$ & Medio & $35.72(6.00)$ & Medio & $47(8.64)$ & Alto \\
AR & $39.86(8.64)$ & Medio & $36.95(6.60)$ & Medio & $42.77(9.58)$ & Medio \\
DE & $42.70(6.22)$ & Medio & $40.72(4.60)$ & Medio & $44.68(7.07)$ & Medio \\
DR & $47.47(6.70)$ & Alto & $45.45(5.73)$ & Alto & $49.5(7.10)$ & Alto \\
EyM & $26.61(8.15)$ & $12.90 \%^{*}$ & $25.86(6.08)$ & $12.07 \%^{*}$ & $27.36(9.90)$ & $13.73 \%^{*}$ \\
CDD & $23.5(8.27)$ & $15.97 \%^{*}$ & $19.9(4.60)$ & $10.98 \%^{*}$ & $27.09(9.59)$ & $20.95 \%^{*}$ \\
AyP & $7.88(3.51)$ & $16.19 \%^{*}$ & $6.4(1.62)$ & $10.03 \%^{*}$ & $9.36(4.24)$ & $22.34 \%^{*}$ \\
SS & $8.31(2.70)$ & $11.06 \%^{*}$ & $7.63(1.73)$ & $8.78 \%^{*}$ & $9(3.32)$ & $13.33 \%^{*}$ \\
FS & $4.61(1.76)$ & $8.96 \%^{*}$ & $5.09(1.74)$ & $11.61 \%^{*}$ & $4.13(1.69)$ & $6.31 \%^{*}$ \\
PT & $70.93(20.82)$ & $13.64 \%^{*}$ & $64.9(13.10)$ & $11.07 \%^{*}$ & $76.95(25.30)$ & $16.21 \%^{*}$ \\
\hline & & & & & & \\
\hline
\end{tabular}

Nota: $\mathrm{AE}=$ Ansiedad Estado, $\mathrm{AR}=\mathrm{Ansiedad}$ Rasgo, $\mathrm{DE}=$ Depresión Estado, $\mathrm{DR}=$ Depresión Rasgo, EyM=Energía y movilidad en calidad de vida, $C D D=C o n t r o l$ de la diabetes en la calidad de vida, AyP=ansiedad y preocupación en calidad de vida, SS=Sobrecarga social en calidad de vida, FS=Funcionamiento sexual en calidad de vida, PT=Total de afectación de la calidad de vida, "Equivalente a (\%) de afectación.

Como se observa en la Figura 1, los análisis t de Student mostraron que los fumadores mostraron mayores niveles estadísticamente significativos en comparación con los no fumadores, con respecto a la ansiedad como estado y rasgo $(t(42)=5.02, p=.000,95 \%$ IC de 6.74 a 15.80 ; $t(37.26)=2.34, p=.024,95 \%$ IC de .79 a 10.84 respectivamente), depresión como estado y rasgo $(t(36.06)=2.19, p=.034,95 \%$ IC de .30 a $7.60 ; t(42)=2.07, p=.044,95 \%$ IC de .114 a 7.97 respectivamente), y las sub escala del D-39 de $\operatorname{CDD}(t(42)=3.16, p=.003,95 \%$ IC de 2.60 a 11.76$)$ y ansiedad y preocupación $(t(42)=3.04, p=.004,95 \%$ IC de .99 a 4.91). No se observaron diferencias estadísticamente significativas con respecto al resto de las sub escalas ni el total de calidad de vida.

De acuerdo con los objetivos específicos, y a partir de los análisis de correlación de Pearson tomando en cuenta solo a aquellos fumadores, se observó que la sub escala AyP del D-39 se correlacionó estadísticamente significativamente con el patrón de consumo ( $r=.487, p=.021)$, así como la dependencia a la nicotina $(r=.608, p=.003)$ con el patrón de consumo. Por lo tanto, los análisis siguientes se realizaron sólo con las sub escalas que se encontraron correlacionadas como variables predictoras. 

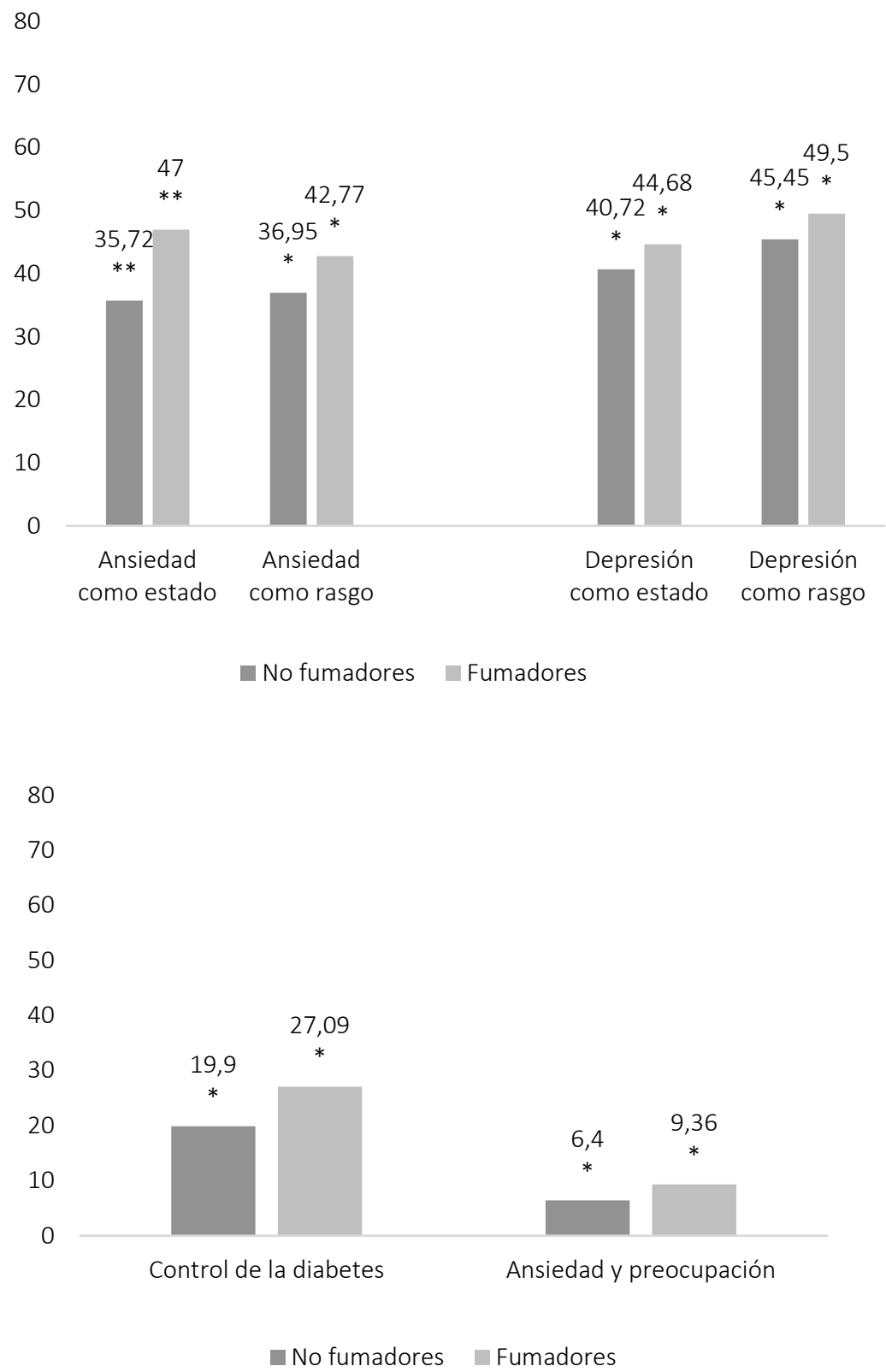

Figura 1. Arriba se muestra áreas de ansiedad y depresión donde se observaron diferencias estadísticamente significativas. Abajo se muestra las sub escalas del D-39 donde se encontraron diferencias estadísticamente significativas. Estos análisis se realizaron entre fumadores $(n=22)$ y no fumadores $(n=22)$ con DM2. Puntuación media en eje Y.

*Estadísticamente significativo al nivel $p<.05 .{ }^{* *}$ Estadísticamente significativo al nivel $p<.000$.

Con respecto a los ocho modelos de análisis de regresión lineal múltiple que se realizaron para cumplir con los objetivos específicos, se observó que a pesar de que todos los modelos analizados resultaron estadísticamente significativos (todas las $\mathrm{p}<.05$ ), no en todos ellos, las variables moderadoras fueron significativas. Específicamente, en tres de estos modelos se observó que las variables que demostraron ser moderadores estadísticamente significativas de la asociación entre la sub escala AyP y el patrón de consumo, fueron la ansiedad como rasgo $(F(3)=6.134, p=.005)$, la depresión como estado $(F(3)=5.410, p=.008)$, y la depresión como rasgo $(F(3)=8.659, p=.001)$, mientras que en sólo uno de los ocho modelos originales se observó que la 
variable que modera la relación entre la sub escala de AyP y el nivel de dependencia a la nicotina fue la depresión como estado $(F(3)=4.45, p=.017)$. En las Tablas 3 a la 6 se muestran los detalles de los modelos en donde las variables moderadoras resultaron estadísticamente significativas.

Tabla 3

Modelo de regresión lineal múltiple entre ansiedad y preocupación en calidad de vida y patrón de consumo con ansiedad como rasgo como moderador

\begin{tabular}{lccccccc}
\hline$F(g)$ Varianza & $\begin{array}{c}\text { Vexplicada } \\
\text { ajustada }\end{array}$ & $p$ & $B$ & $t$ & $p$ & $95 \%$ IC para $B$ \\
\hline $6.134(3)$ & $50.6 \%$ & $.005^{*}$ & .073 & .372 & .714 & -.341 & .487 \\
& & & -4.83 & -2.275 & $.035^{*}$ & -9.297 & -.370 \\
& & & .432 & 2.772 & $.013^{*}$ & .104 & .759 \\
\hline
\end{tabular}

${ }^{*} p<.05, n=22$.

Tabla 4

Modelo de regresión lineal múltiple entre ansiedad y preocupación en calidad de vida y patrón de consumo con depresión como estado como moderador

\begin{tabular}{|c|c|c|c|c|c|c|c|}
\hline$F(g l)$ & $\begin{array}{l}\text { Varianza } \\
\text { explicada } \\
\text { ajustada }\end{array}$ & $p$ & $B$ & $t$ & $p$ & \multicolumn{2}{|c|}{$95 \%$ IC para $B$} \\
\hline \multirow{3}{*}{$5.410(3)$} & \multirow{3}{*}{$47.4 \%$} & \multirow{3}{*}{$.008^{*}$} & .458 & .951 & .354 & -.553 & 1.469 \\
\hline & & & -.278 & -1.003 & .329 & -.859 & .304 \\
\hline & & & 3.759 & 2.839 & $.011^{*}$ & .978 & 6.541 \\
\hline
\end{tabular}

Tabla 5

Modelo de regresión lineal múltiple entre ansiedad y preocupación en calidad de vida y patrón de consumo con depresión como estado como moderador

\begin{tabular}{lccccccc}
\hline$F(g l)$ & $\begin{array}{c}\text { Varianza } \\
\text { explicada } \\
\text { ajustada }\end{array}$ & $p$ & $B$ & $t$ & $p$ & $95 \%$ IC para $B$ \\
\hline $8.659(3)$ & $52.2 \%$ & $.001^{*}$ & -.433 & -1.448 & 1.65 & -1.061 & .195 \\
& & & .245 & .581 & .569 & -.643 & 1.134 \\
& & & 4.783 & 3.673 & $.002^{*}$ & 2.047 & 7.518 \\
\hline${ }^{*} p<05, n=22$
\end{tabular}

${ }^{\circ} p<.05, n=22$. 
Tabla 6

Modelo de regresión lineal múltiple entre ansiedad y preocupación en calidad de vida y dependencia a la nicotina con depresión como estado como moderador

\begin{tabular}{|c|c|c|c|c|c|c|c|}
\hline$F(g l)$ & $\begin{array}{l}\text { Varianza } \\
\text { explicada }\end{array}$ & $p$ & $B$ & $t$ & $p$ & \multicolumn{2}{|c|}{$95 \%$ IC para $B$} \\
\hline \multirow{3}{*}{$5.884(3)$} & \multirow{3}{*}{$49.5 \%$} & \multirow{3}{*}{$.006^{*}$} & 1.214 & 2.020 & .059 & -.049 & 2.477 \\
\hline & & & -.328 & -.950 & .355 & -1.055 & .398 \\
\hline & & & 3.503 & 2.118 & $.048^{*}$ & .027 & 6.978 \\
\hline
\end{tabular}

${ }^{*} p<.05, n=22$.
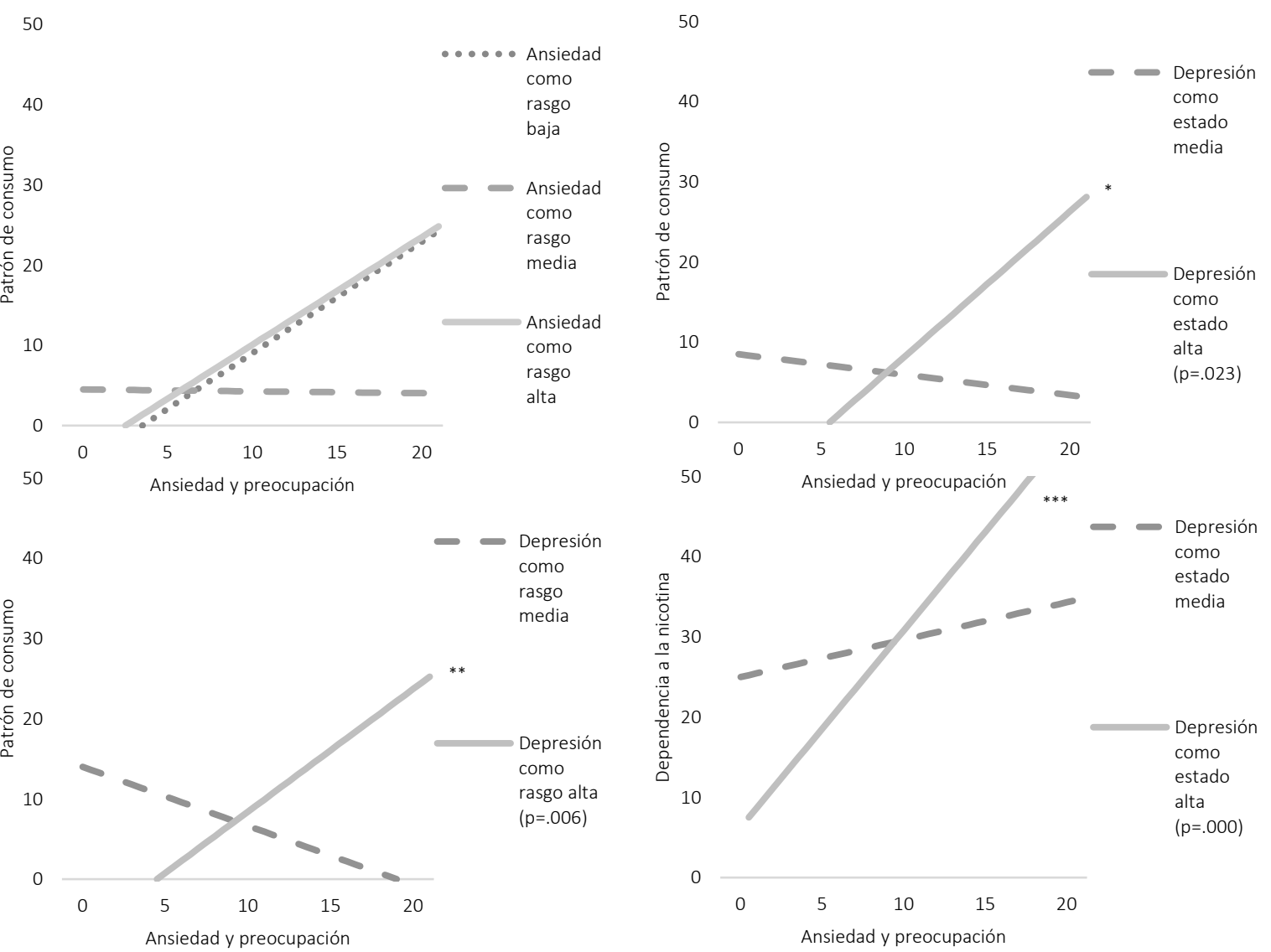

Figura 2. Resultados de los análisis de regresión lineal simples de la sub escala AyP como predictora del patrón de consumo y nivel de dependencia de acuerdo con los niveles de ansiedad y depresión.

${ }^{*}$ Estadísticamente significativo al nivel $p<.05 .{ }^{* *}$ Estadísticamente significativo al nivel $p<.01$. ${ }^{* * *}$ Estadísticamente significativo al nivel $p<.000$. 
De acuerdo con los niveles de depresión y ansiedad como rasgo y estado de aquellos fumadores con DM2, se observó que no hubo quienes presentaran ansiedad como estado baja, el $36.4 \%(n=8)$ tuvieron ansiedad como estado media y $63.6 \%(n=14)$ alta. El $13.6 \%(n=3)$ tuvieron ansiedad como rasgo baja, el $31.8 \%(n=7)$ media, y el $54.5 \%(n=12)$ alta. Con respecto a la depresión, no hubo quienes presentaran depresión como estado baja, el 50\% ( $n=11)$ tuvieron depresión como estado media, y el 50\% ( $n=11)$ alta. Tampoco hubo quienes presentaran depresión como rasgo baja, el $22.7 \%(n=5)$ tuvieron depresión como rasgo media, y el $77.3 \%$ $(n=17)$ alta.

Con respecto a los análisis de regresión simple que se realizaron con la sub escala AyP y patrón de consumo, de acuerdo con los niveles de ansiedad y depresión como rasgo y estado para cumplir con el último objetivo específico, se encontró que la sub escala AyP logra predecir significativamente al patrón de consumo cuando el nivel de depresión como estado es alto $(F(1)=7.490, p=.023, B=1.857,95 \%$ IC de .322 a 3.391$)$ con una varianza explicada del $67.4 \%, y$ cuando el nivel de depresión como rasgo es alto $(F(1)=10.416, p=.006, B=1.583,95 \%$ IC de .537 a 2.628) con una varianza explicada del $43.3 \%$. Asimismo, la sub escala AyP logra predecir al nivel de dependencia a la nicotina cuando el nivel de depresión como estado es alto $(F(1)=28.781$, $p<.000, B=2.509,95 \%$ IC de 1.451 a 3.566) con una varianza explicada del $87.3 \%$ (ver Figura 2).

\section{Discusión}

El objetivo general de este estudio fue evaluar las diferencias que existen entre fumadores y no fumadores con DM2, con respecto a la ansiedad, la depresión en sus dimensiones rasgo y estado, y la calidad de vida. Con respecto a esto, los resultados mostraron que las personas con diabetes que consumían tabaco tuvieron puntuaciones estadísticamente significativas más altas en ansiedad y depresión como rasgo y estado, comparadas con quienes no fumaban. Estos resultados coinciden con la literatura, donde se ha mostrado que algunas personas fumadoras con DM presentan mayor sintomatología de ansiedad y depresión con respecto a quienes tienen DM y no fuman (Osme et al., 2012). Es importante resaltar que se observaron niveles medios tanto en depresión como ansiedad, exceptuando a la depresión como rasgo, que tuvo un nivel alto para todos los participantes, y la ansiedad como estado que fue alta sólo para los fumadores. Estas diferencias en la sintomatología de ansiedad y depresión entre fumadores y no fumadores son de gran importancia a tomar en cuenta al diseñar intervenciones para el cese de consumo en personas con DM, ya que se han considerado como factores del mantenimiento de fumar en quienes tienen DM (Coster, Gulliford, Seed, Powrie, \& Swaminathan, 2000; Fabián \& Cobo, 2007; Karter, et al., 2001). Además, debido a la alta prevalencia de sintomatología de ansiedad (Alharithy, Abdalgader, Alobaylan, Alsugair, \& Alswat, 2019; Alzahrani, Alghamdi, Algarni, Alshareef, \& Alzahrani, 2019; Al-Mohaimeed, 2017; Dehesh, Dehesh, \& Shojaei, 2020; Khalighi et al., 2019) y sintomatología de depresión (Alzahrani et al., 2019; Al-Mohaimed, 2017; Dehesh et al., 2020; Khalighi et al., 2019; Roy \& Lloyd, 2012; Tovilla-Zárate et al., 2012) en personas con DM, se recomienda que la reducción de la sintomatología de ansiedad y depresión se incluyan en el manejo estándar de la DM (ADA, 2020; Nicolucci et al., 2013; Peyrot \& Rubin, 2007)

Por otro lado, con respecto a la calidad de vida, sólo en dos de las sub escalas se encontraron diferencias estadísticamente significativas (Control De la Diabetes, y Ansiedad y Preocupación), en donde las personas fumadoras con DM2 presentaron mayores puntuaciones con respecto a aquellas personas no fumadoras con DM2. Esto es, las personas con diabetes que fumaban tuvieron menor calidad de vida, específicamente en las áreas que se relacionan con aspectos con 
los cuidados de la enfermedad, además de que presentan mayores preocupaciones y ansiedad asociada a la DM2. Con respecto al resto de sub escalas que evalúan la calidad de vida por la DM2 y del total de la escala, no se encontraron diferencias estadísticamente significativas, aunque de forma general, se observa que en todas las sub escalas (exceptuando el área de Funcionamiento Sexual) y total, los porcentajes de afectación en la calidad de vida son mayores en aquellas personas que consumen tabaco. Se ha encontrado en la literatura que aquellas personas con DM tienen una mayor afectación en la calidad de vida (Akinci et al., 2008; Kalda et al., 2008; Oliveira et al., 2017), y el consumo de tabaco también puede hacer que la calidad de vida de las personas sea menor (Castro, Matsuo, \& Nunes, 2010; de Miguel Díez et al., 2010; Džubur et al., 2014; López-Nicolás et al., 2018), lo que hace que al combinar el consumo de tabaco con la DM, la calidad de vida se vea afectada de mayor manera (Ahmed et al., 2018; Alencar et al., 2019; Mokhtari et al., 2019; Oliveira et al., 2017). Por lo que los resultados de este estudio concuerdan con la literatura, específicamente con respecto a que se observa una mayor afectación en la calidad de vida de las personas fumadoras con DM2, en comparación con quienes no fuman y tienen DM2, lo cual resalta la importancia del diseño de intervenciones para el cese de consumo de tabaco en personas con DM que haga uso de técnicas que impacten en la calidad de vida, ya que como se ha visto, al consumir tabaco y padecer DM, esta área se ve más afectada.

Como objetivos específicos, se planteó evaluar si la ansiedad y depresión como rasgo y estado moderan la asociación entre la calidad de vida y el consumo de tabaco en personas fumadoras, así como evaluar el nivel de predicción que tiene la calidad de vida con respecto al consumo de tabaco, de acuerdo con los niveles de ansiedad y depresión en fumadores con DM2. En todos los modelos de regresión que se probaron con aquellas personas fumadoras con DM2, donde se incluye a la puntuación de la sub escala de Ansiedad y Preocupación de calidad de vida y a la sintomatología de ansiedad o depresión como variables predictoras, y al patrón de consumo o al nivel de dependencia como variables dependientes, se observa que la puntuación de la sub escala de Ansiedad y Preocupación logra predecir tanto al patrón de consumo y al nivel de dependencia a la nicotina. A pesar de esto, no en todos los modelos la ansiedad o depresión lograron moderar esta asociación. Específicamente, las variables que resultaron ser moderadoras de la asociación entre la sub escala de Ansiedad y Preocupación y patrón de consumo, fueron la ansiedad como rasgo y la depresión en sus dos dimensiones. Esto muestra que, además de que al aumentar la puntuación con respecto a la sub escala de Ansiedad y Preocupación, es más probable que el patrón de consumo de tabaco sea mayor, el aumentar la sintomatología de ansiedad como rasgo, o la depresión, ya sea en su forma de rasgo o estado, resulta en aún mayor probabilidad de consumo de tabaco. Se puede observar una asociación similar con respecto a la sub escala de Ansiedad y Preocupación con respecto a la dependencia a la nicotina, aunque en este caso la única variable que demostró ser moderadora fue la depresión como estado. En la literatura se ha encontrado que las variables de ansiedad y depresión logran predecir al consumo de tabaco en población general (Lira-Mandujano et al., 2018; Lira-Mandujano, Valdez-Piña, PérezGonzález, Pech-Puebla, \& Arriaga-Ramírez, 2020), lo cual concuerda con los resultados de este estudio, aunque es necesaria más investigación acerca del nivel de predicción y la capacidad de predicción y moderación de estas variables en personas fumadoras con DM2.

Por último, al analizar el nivel de predicción de la sub escala de Ansiedad y Preocupación con respecto al patrón de consumo y nivel de dependencia de acuerdo con los niveles de ansiedad y depresión como rasgo y estado, se observó que la sub escala de Ansiedad y Preocupación logró predecir al patrón de consumo sólo en aquellas personas que tuvieron un nivel de depresión alto, tanto en rasgo como estado. Además de que la sub escala de Ansiedad y Preocupación logra 
predecir al nivel de dependencia a la nicotina sólo cuando el nivel de depresión como estado es alto. En estas asociaciones, al aumentar la puntuación de la sub escala de Ansiedad y Preocupación, la probabilidad tanto de patrón de consumo como dependencia a la nicotina es mayor. Es importante resaltar que en todas estas asociaciones que resultaron estadísticamente significativas, el porcentaje de varianza explicada fue de moderado a alto. Debido a que estas asociaciones se observaron en mayor medida en personas con niveles de depresión altos, se resalta la importancia de continuar estudiando las complejas asociaciones que existen entre estas variables, ya que es un campo poco estudiado en la literatura y que es necesario a tomar en cuenta para el diseño de intervenciones para dejar de fumar en personas con DM más efectivas.

El presente estudio proporciona información acerca de la diferencia que existe entre la ansiedad y depresión como rasgo y estado, y no sólo la sintomatología de ansiedad y depresión en la última semana. Con respecto a esto, se observa que las dimensiones que tuvieron mayor impacto con respecto a las asociaciones evaluadas fue la de depresión como estado, la cual se refiere a la forma en que la persona se siente al momento de la evaluación, y la ansiedad como rasgo, que se refiere a la forma en que la persona se siente de forma en general. Estos resultados resaltan la importancia de tomar en cuenta los aspectos emocionales no sólo en el momento de la evaluación sino como un rasgo, ya que se pueden encontrar diferencias que tienen relevancia al diseñar intervenciones para dejar de fumar.

\section{Limitaciones}

En la interpretación de los resultados del presente estudio se deben considerar los siguientes puntos: a) el patrón de consumo y el nivel de dependencia fueron de bajos a medios, lo cual hace que los resultados puedan ser generalizables sólo a poblaciones con las mismas características, b) los puntajes correspondientes a la afectación en la calidad de vida por la DM en la mayoría de las sub escalas (excepto en las sub escalas de Control De la Diabetes, y Ansiedad y Preocupación), así como en el total de la escala fue baja, lo que puede deberse a que la población estudiada no percibe que la DM se encuentre afectando a su calidad de vida, al menos en esta etapa de la enfermedad, al tratarse de pacientes ambulatorios y con un control adecuado de la DM, y esto podría haber causado un sesgo con respecto a las asociaciones resultantes, imposibilitando el análisis cuando las personas tienen una mayor afectación en la calidad de vida, y c) en cuanto a los objetivos específicos, no se encontraron personas con niveles de ansiedad estado bajos, ni depresión bajos, tanto en rasgo y estado, y en general, hubo pocas personas en algunos de los demás grupos, lo que limita los análisis de datos a personas con estas características.

Por todo esto, se sugiere que para futuras investigaciones y debido a la poca variabilidad en las variables estudiadas: a) que se incluya a un mayor número de participantes, se incluyan personas con b) diferentes niveles de dependencia a la nicotina, para poder realizar análisis más específicos de acuerdo con estos niveles, con el fin de evaluar si el tener diferentes niveles de dependencia influye en la asociación evaluada, c) con diferentes niveles de calidad de vida, con el fin de evaluar si diferentes niveles de afectación en la calidad de vida se asocia con el consumo de tabaco, d) con diferentes niveles de ansiedad y depresión como rasgo y estado, además de que los grupos de comparación sean homogéneos, con el fin de realizar un análisis más sensible con cada uno de estos grupos, asimismo, se sugiere e) evaluar si una o más de las variables sociodemográficas como la edad o el género, alguna de las variables asociadas con la historia de consumo como la edad de inicio de consumo de tabaco o los años de consumo regular, o variables asociadas con la historia de la diabetes como los años con el diagnóstico o el nivel de glucosa, 
juegan un papel en la asociación entre ansiedad, depresión y calidad de vida, con respecto al patrón de consumo y dependencia a la nicotina, y por último, f) continuar con el análisis de los factores que juegan un papel como moderadores en la asociación entre el consumo de tabaco y aquellos factores que han demostrado mantener el consumo de tabaco, ya que este campo se encuentra poco estudiado.

\section{Conclusiones}

Este estudio aporta información relevante y novedosa acerca de la asociación y función moderadora que existe entre la ansiedad, depresión y calidad de vida con respecto al consumo de tabaco en personas fumadoras y no fumadoras con DM2, el cual es un campo poco estudiado en la literatura científica, y muy necesario para la comprensión de los factores que afectan a las personas consumidoras de tabaco con DM, así como para el diseño de intervenciones efectivas para el cese de consumo de tabaco en personas con DM, además de funcionar como base para futuras investigaciones que estudien a esta población. Se puede concluir en este estudio que las personas fumadoras con DM2 tuvieron más síntomas de depresión y ansiedad, tanto en rasgo como estado, además de menor calidad de vida por tener DM2 (específicamente en las áreas de Control De la Diabetes, y Ansiedad y Preocupación), con respecto a quienes tuvieron DM2, pero no consumen tabaco. Además, en quienes consumían tabaco, la Ansiedad y Preocupación en la calidad de vida predice al patrón de consumo y al nivel de dependencia, y específicamente, la ansiedad como rasgo y la depresión en sus dos dimensiones moderan la asociación entre la Ansiedad y Preocupación en calidad de vida y patrón de consumo, mientras que la depresión como estado modera la asociación entre la Ansiedad y Preocupación en la calidad de vida y dependencia a la nicotina. Específicamente, la ansiedad y depresión moderan la relación entre la calidad de vida y el consumo de tabaco (patrón de consumo y dependencia a la nicotina) en personas mexicanas fumadoras con DM2, en donde a mayor afectación a la calidad de vida, mayor sintomatología de ansiedad y mayor sintomatología de depresión, aumenta la dependencia a la nicotina y el patrón de consumo.

Es importante resaltar la necesidad de prevenir en primer lugar la DM, al promover la salud en general de la población, y cuando se presente un diagnóstico de esta enfermedad es necesario evaluar si existe o no consumo de tabaco, con el fin de que realizar una canalización a un programa para dejar de fumar que sea diseñado particularmente para personas con DM.

Por otro lado, debido a que la DM afecta la calidad de vida de las personas, es necesario atender la salud mental de las personas con esta enfermedad, y no sólo enfocar el tratamiento de la DM a los cuidados médicos y cambios de estilo de vida, ya que como se vio en este estudio, existen consecuencias con respecto a la sintomatología de ansiedad, depresión y calidad de vida cuando hay un diagnóstico de DM y más aún cuando hay consumo de tabaco.

Por lo tanto, no sólo se recomienda el diseño de intervenciones para dejar de fumar en personas con DM, sino que es importante que se incluyan componentes y técnicas cognitivoconductuales que impacten en las áreas de calidad de vida y en la sintomatología de ansiedad y depresión, como técnicas de respiración, reestructuración cognitiva, y técnicas de autocontrol.

\section{Conflicto de intereses}

Los autores declaran no tener algún conflicto de intereses. 


\section{Agradecimientos}

Se agradece al Consejo Nacional de Ciencia y Tecnología (CONACYT) por otorgar una beca al primer autor para realizar estudios de doctorado en el Programa de Doctorado de Psicología de la Universidad Nacional Autónoma de México (UNAM), con número de CVU/Becario: $620328 / 336016$.

\section{Referencias}

Ahmed, N., Tomalika, N., Rhaman, M. M., Momtaz, H., \& Haque, M. (2018). Quality of life in patients with diabetes mellitus. IMC Journal of Medical Science, 12(2), 1-8. doi:10.3329/imcjms.v12i2.39666

Akinci, F., Yildirim, A., Gözu, H., Sargini, H., Orbay, E., \& Sargin, M. (2008). Assesment of healthrelated quality of life (HRQoL) of patients with type 2 diabetes in Turkey. Diabetes Research and Clinical Practice, 79(1), 117-123. doi:10.1016/j.diabres.2007.07.003

Alencar, D. C., Lages, P. F., Neiva, M. J. L. M., Alencar, A. M. P. G., Moreira, W. C., \& Ibiapina, A. R. S. (2019). Dimensions of the quality of life negatively affected in people living with Diabetes Mellitus. Revista de Pesquisa: Cuidado é Fundamental Online, 11(1), 199-204. doi:10.9789/2175-5361.2019.v11i1.239-244

Albaroodi, K. A., Syed, S. S., Shafie, A. A., Awaisu, A., \& Lajis, R. (2014). The prevalence of tobacco smoking in patients with diabetes in hospital Paulau Pinang, Malasya. Value in Health, 17, A323-A686. doi:10.1016/j.jval.2014.08.2023

Alharithy, M. K., Abdalgader, O. R., Alobaylan, M. M., Alsugair, Z. O., \& Alswat, K. A. (2019). Anxiety prevalence in type 2 diabetes patients in Taif Saudi Arabia. Saudi Journal for Health Sciences, 8(2), 88-92. doi:10.4103/sjhs.sjhs 13318

Alzahrani, A., Alghamdi, A., Alqarni, T., Alshareef, R., \& Alzahrani, A. (2019). Prevalence and predictors of depression, anxiety, and stress symptoms among patients with type II diabetes attending primary healthcare centers in the western region of Saudi Arabia: a cross-sectional study. International journal of mental health systems, 13, 48. doi:10.1186/s13033-019-0307-6

Al-Mohaimeed A. A. (2017). Prevalence and factors associated with anxiety and depression among type 2 diabetes in Qassim: A descriptive cross-sectional study. Journal of Taibah University Medical Sciences, 12(5), 430-436. doi:10.1016/i.jtumed.2017.04.002

American Diabetes Association (2018). Standards of Medical Care in Diabetes-2018. Diabetes Care, 41(1), S38-S50. doi:10.2337/dc18-S004

American Diabetes Association (2020). 5. Facilitating behavior change and well-being to improve health outcomes: Standards of Medical Care in Diabetes-2020. Diabetes Care, 43 Suppl 1, S48-S65. doi:10.2337/dc20-S005

Boyer, J., \& Earp, J. (1997). The development of an instrument for assessing the quality of life of people with diabetes: Diabetes-39. Medical Care, 35(5), 440-453. doi:10.1097/00005650-199705000-00003 
Castro, M. R., Matsuo, T., \& Nunes, S. O. (2010). Clinical characteristics and quality of life of smokers at a referral center for smoking cessation. Jornal brasileiro de pneumologia, 36(1), 67-74. doi:10.1590/s1806-37132010000100012

Centers for Disease Control and Prevention (2017). How tobacco Smoke Causes Disease. Recuperado https://www.cdc.gov/tobacco/data statistics/sgr/2010/consumer booklet/pdfs/consu mer.pdf

Coster, S., Gulliford, M. C., Seed, P. T., Powrie, J. K., \& Swaminathan, R. (2000). Self-monitoring in Type 2 diabetes mellitus: a meta-analysis. Diabetic medicine, 17(11), 755-761. doi:10.1046/j.1464-5491.2000.00390.x

de Miguel Díez, J., Esteban, M. M., Puente, L., Hernández, V., Carrasco, P., Alvarez-Sala, L. A., \& Jiménez, R. (2010). Relationship Between Tobacco Consumption and Health-related Quality of Life in Adults Living in a Large Metropolitan Area. Lung, 188, 393-399. doi:10.1007/s00408-010-9256-1

Dehesh T, Dehesh P, \& Shojaei S. (2020). Prevalence and Associated Factors of Anxiety and Depression Among Patients with Type 2 Diabetes in Kerman, Southern Iran. Diabetes, Metabolic Syndrome and Obesity: Targets and Therapy, 13, 1509-1517. doi: $\underline{10.2147 / D M S O . S 249385}$

Dirección General de Epidemiología (2018). Distribución de casos nuevos de enfermedad por grupos de edad Estados Unidos Mexicanos 2018. Población General. Secretaría de Salud. Recuperado de http://www.epidemiologia.salud.gob.mx/anuario/2018/morbilidad/nacional/distribucio n casos nuevos enfermedad grupo edad.pdf

Džubur, A., Mehić, B., Džubur, A., Filipovska-Mušanović, M., Denjalić, A., \& Hasanbegović, I. (2014). Quality of life for tobacco smokers in relation to their socioeconomic status. Medicinski glasnik, 11(1), 210-215.

Etter, J. F., Le Houezec, J., \& Perneger, T. V. (2003). A self-administered questionnaire to measure dependence on cigarettes: the cigarette dependence scale. Neuropsychopharmacology, 28, 359-370. doi:10.1038/sj.npp.1300030

Fabián, M. G., \& Cobo, A. C. (2007). Tabaquismo y diabetes. Revista del Instituto Nacional de Enfermedades Respiratorias, 20(2), 149-158.

Galicia, C. S. T., \& Segundo, H. P. (2007). Validación del inventario de ansiedad rasgo-estado (IDARE) de Spielberger en adolescentes estudiantes de secundaria mexicanos de la delegación Xochimilco (Tesis de licenciatura). Recuperado de http://132.248.9.195/pd2007/0617640/Index.html

Gilliani, A. H., Aziz, M. M., Bashir, S., Mustafa, A., \& Fang, Y. (2017). Evaluation of Smoking Prevalence and its associated factors among the diabetics in Pakistan. Value in Health, 20(9), A853-A943. doi:10.1016/i.jval.2017.08.2863 
Grandy, S., Chapman, R. H., Fox, K. M., \& SHIELD Study Group (2008). Quality of life and depression of people living with type 2 diabetes mellitus and those at low and high risk for type 2 diabetes: findings from the Study to Help Improve Early evaluation and management of risk factors Leading to Diabetes (SHIELD). International journal of clinical practice, 62(4), 562-568. doi:10.1111/j.1742-1241.2008.01703.x

Huo, L., Shaw, J. E., Wong, E., Harding, J. L., Peeters, A., \& Magliano, D. J. (2016). Burden of diabetes in Australia: life expectancy and disability-free life expectancy in adults with diabetes. Diabetología, 59, 1437-1445. doi:10.1007/s00125-016-3948-x

Instituto Nacional de Salud Pública (2016). Encuesta Nacional de Salud y Nutrición de Medio Camino 2016 Informe Final de Resultados. Recuperado de https://www.gob.mx/cms/uploads/attachment/file/209093/ENSANUT.pdf

International Diabetes Federation (2012). Global Guideline for Type 2 Diabetes. Recuperado de https://www.idf.org/e-library/guidelines/79-global-guideline-for-type-2-diabetes

International Diabetes Federation (2017). IDF Diabetes Atlas English edition 2017. Recuperado de https://www.diabetesatlas.org/en/resources/?gclid=EAlalQobChMInqfOKSE5WIVIf jBX2IXWGIEAAYASAAEgJZ6 D BWE

Kalda, R., Rätsep, A., \& Lember, M. (2008). Predictors of quality of life of patients with type 2 diabetes. Patient preference and adherence, 2, 21-26.

Karter, A. J., Ackerson, L. M., Darbinian, J. A., D'Agostino, R. B., Jr, Ferrara, A., Liu, J., \& Selby, J. V. (2001). Self-monitoring of blood glucose levels and glycemic control: the Northern California Kaiser Permanente Diabetes registry. The American journal of medicine, 111(1), 1-9. doi:10.1016/s0002-9343(01)00742-2

Khalighi, Z., Badfar, G., Mahmoudi, L., Soleymani, A., Azami, M., \& Shohani, M. (2019). The prevalence of depression and anxiety in Iranian patients with diabetes mellitus: A systematic review and meta-analysis. Diabetes \& metabolic sindrome: Clinical research \& Reviews, 13(4), 2785-2794. doi:10.1016/j.dsx.2019.07.004

Lira-Mandujano, J. (2009). Programa de intervención breve motivacional para fumadores y terapias de sustitución con nicotina: Resultados de un ensayo clínico aleatorio controlado (Tesis doctoral). Recuperado de https://ru.dgb.unam.mx/handle/DGB UNAM/TES01000639778

Lira-Mandujano, J., Valdez-Piña, E., Núñez-Lauriano, M., Pech-Puebla, D, Morales-Chainé, S., \& Cruz-Morales, S. (2018). Ansiedad, depresión, afecto negativo y positivo asociados con el consumo de tabaco en universitarias. Revista Internacional de Investigación en Adicciones, 4(1), 4-12. doi:10.28931/riiad.2018.1.02

Lira-Mandujano, J., Valdez-Piña, E., Pérez-González, M. A., Pech-Puebla, D., \& Arriaga-Ramírez, J. C. P. (2020). Asociación entre consumo de tabaco, factores emocionales y motivos para fumar en universitarios. Enseñanza e Investigación en Psicología, 2(1), 47-59. 
López-Carmona, J. M., \& Rodríguez-Moctezuma, R. (2006). Adaptación y validación del instrumento de calidad de vida Diabetes 39 en pacientes mexicanos con diabetes mellitus tipo 2. Salud pública de México, 48 (3), 200-211.

López-Nicolás, A., Trapero-Bertran, M., \& Muñoz, C. (2018). Smoking, health-related quality of life and economic evaluation. The European Journal of Health Economics, 19, 747-756. doi:10.1007/s10198-017-0919-1

Maric-Bilkan, C. (2017). Sex differences in micro- and macro-vascular complications of diabetes mellitus. Clinical Science, 131, 833-846. doi:10.1042/CS20160998

Martín, M., Grau, J. A., Ramírez, V., \& Grau, R. (2001). El Inventario de Depresión Rasgo-Estado (IDERE): Desarrollo y Potencialidades. Revista Psicología.com, 5(1). Recuperado de http://www.psiquiatria.com/diagnostico47/el-inventario-de-depresion-rasgo-estadoidere-desarrollo-y-potencialidades

Martín-Carbonell, M., Riquelme-Marín, A., Ortigosa-Quiles, J. M., Meda, R. M., Córdova, A. M., Enríquez-Santos, J. A., \& Pérez-Díaz, R. (2012). Utilidad del Inventario de Depresión Rasgo-Estado (IDERE) para el diagnóstico clínico. Un estudio con muestras de cuatro países iberoamericanos. Anales de Psicología, 28(3), 763-771. doi:10.6018/analesps.28.3.133711

Mokhtari, Z., Gheshlagh, R. G., \& Kurdi, A. (2019). Health-related quality of life in Iranian Patients with type 2 diabetes: An updated meta-analysis. Diabetes \& Metabolic Syndrome: Clinical Research \& Reviews, 13, 402-407. doi:10.1016/j.dsx.2018.10.007

National Institute on Drug Abuse (2019). Cigarettes and Other Tobacco Products. Recuperado de https://www.drugabuse.gov/publications/drugfacts/cigarettes-other-tobacco-products

Nicolucci, A., Kovacs Burns, K., Holt, R. I., Comaschi, M., Hermanns, N., Ishii, H., ... DAWN2 Study Group (2013). Diabetes Attitudes, Wishes and Needs second study (DAWN2 ${ }^{\mathrm{TM}}$ ): crossnational benchmarking of diabetes-related psychosocial outcomes for people with diabetes. Diabetic medicine, 30(7), 767-777. doi:10.1111/dme.12245

Oliveira, B. C., Bomfim, E. S., Ribeiro, I. J. S., Carmo, E. A., Boery, R. N. S. O., \& Boery, E. N. (2017). Factors associated with the quality of life of individuals affected by diabetes mellitus. Revista Baiana de Enfermagem, 31(4), e18247. doi:10.18471/rbe.v31i4.21481

Organización Mundial de la Salud (2017). Diabetes. Recuperado de http://www.who.int/mediacentre/factsheets/fs312/es

Organización Mundial de la Salud (2011). La prueba de detección de consumo de alcohol, tabaco y sustancias (ASSIST). Manual para uso en la atención primaria. Recuperado de https://www.who.int/substance abuse/activities/assist screening spanish.pdf

Osme, S. F., Ferreira, L., Jorge, M. T., de Souza Andréo, J., Jorge, M., de Melo Costa Pinto, R., ... Jorge, P. T. (2012). Difference between the prevalence of symptoms of depression and anxiety in non-diabetic smokers and in patients with type 2 diabetes with and without nicotine dependence. Diabetology \& Metabolic Syndrome, 4 (1), 39-48. doi: $\underline{10.1186 / 1758-5996-4-39}$ 
Ou, H. T., Yang, C. Y., Wang, J. D., Hwang, J. S., \& Wu, J. S. (2016). Life Expectancy and Lifetime Health Care Expenditures for Type 1 Diabetes: A National Longitudinal Cohort of Incident Cases Followed for 14 Years. Value in Health, 19, 976-984. doi:10.1016/j.jval.2016.05.017

Pereira, C. D., Sant'Ana, L. M. D., das Gracas, M. C., Pires, L. S., Braga, K. G., \& Fernandes, A. P. (2016). Diabetes mellitus: the linkage between oxidative stress, inflammation, hypercoagulability and vascular complications. Journal of Diabetes and its Complications, 30, 738-745. doi:10.1016/j.jdiacomp.2015.12.018

Peyrot, M., \& Rubin, R. R. (2007). Behavioral and psychosocial interventions in diabetes: a conceptual review. Diabetes Care, 30(10), 2433-2440. doi:10.2337/dc07-1222

Pizzo, M. R., Matsuo, T., \& Vargas, S. O. (2009). Clinical characteristics and quality of life of smokers at a referral center for smoking cessation. The Journal Brasileiro de Pneumologia, 36(1), 67-74. doi:10.1590/S1806-37132010000100012

Robinson, S.M., Sobell, L. C., Sobell, M. B., \& Leo, G. I. (2014). Reliability of the Timeline Followback for cocaine, cannabis, and cigarette use. Psychology of Addictive Behaviors, 28(1), 154162. doi:10.1037/a0030992

Roy, T., \& Lloyd, E. (2012). Epidemiology of depression and diabetes: A systematic review. Journal of Affective Disorders, 142, S8-S21. doi:10.1016/S0165-0327(12)70004-6

Secretaría de Salud (2015). Protocolo Clínico para el Diagnóstico y Tratamiento de la Diabetes. Recuperado de https://www.gob.mx/salud/documentos/protocolo-clinico-para-eldiagnostico-y-tratamiento-de-la-diabetes

Spielberger, C., \& Díaz, R. (2002). Inventario de Ansiedad: Rasgo-Estado. México: Manual Moderno.

Stoddard, P., He, G., \& Schillinger, D. (2010). Smoking behavior among Hispanic adults with diabetes on the United States-México border: a public health opportunity. Revista Panamericana de Salud Pública, 28 (3), 221-229.

Tovilla-Zárate, C., Juárez-Rojop, I., Peralta J. Y., Jiménez, M. A., Vázquez, S., Bermúdez-Ocaña, D., ... López, N. L. (2012). Prevalence of Anxiety and Depression among Outpatients with Type 2 Diabetes in the Mexican population. PLOS ONE, 7(5): e36887. doi:10.1371/journal.pone.0036887

Tweed, J. O., Hsia, S. H., Lutfy, K., \& Friedman, T. C. (2012). The endocrine effects of nicotine and cigarette smoke. Trends in Endocrinology and Metabolism, 23(7), 334-342. doi:10.1016/j.tem.2012.03.006

U.S. Department of Health and Human Services (2017). The Health Consequences of Smoking-50 Years of Progress. A report of the Surgeon General. Recuperado de http://www.surgeongeneral.gov/library/reports/50-years-of-progress

Valero, K., Marante, D., Torres R., M., Ramírez, G., Cortéz, R., \& Carlini, R. (2012). Complicaciones microvasculares de la diabetes. Revista Venezolana de Endocrinología y Metabolismo, 10(1), 111-137. 
Walker, J., Colhoun, H., Livingstone, S., McCrimmon, R., Petrie, J., Sattar, N., ... Scottish Diabetes Research Network Epidemiology Group (2018). Type 2 diabetes, socioeconomic status and life expectancy in Scotland (2012-2014): a population-based observational study. Diabetología, 61, 108-116. doi:10.1007/s00125-017-4478-x

World Health Organization (2018). Diabetes. Recuperado de https://www.who.int/newsroom/fact-sheets/detail/diabetes 